\title{
Gravitational wave signatures of the absence of an event horizon. II. Extreme mass ratio inspirals in the spacetime of a thin-shell gravastar
}

\author{
Paolo Pani, ${ }^{1, *}$ Emanuele Berti, ${ }^{2,3, \dagger}$ Vitor Cardoso, ${ }^{2,4,+}$ Yanbei Chen, ${ }^{3,8}$ and Richard Norte ${ }^{3, \|}$ \\ ${ }^{1}$ Dipartimento di Fisica, Università di Cagliari, and INFN sezione di Cagliari, Cittadella Universitaria 09042 Monserrato, Italy \\ ${ }^{2}$ Department of Physics and Astronomy, The University of Mississippi, University, Mississippi 38677-1848, USA \\ ${ }^{3}$ Theoretical Astrophysics 350-17, California Institute of Technology, Pasadena, California 91125, USA \\ ${ }^{4}$ Centro Multidisciplinar de Astrofísica-CENTRA, Department de Física, Instituto Superior Técnico, Av. Rovisco Pais 1, \\ 1049-001 Lisboa, Portugal \\ (Received 25 January 2010; published 5 April 2010)
}

\begin{abstract}
We study gravitational wave emission from the quasicircular, extreme mass ratio inspiral of compact objects of mass $m_{0}$ into massive objects of mass $M \gg m_{0}$ whose external metric is identical to the Schwarzschild metric, except for the absence of an event horizon. To be specific we consider one of the simplest realizations of such an object: a nonrotating thin-shell gravastar. The power radiated in gravitational waves during the inspiral shows distinctive peaks corresponding to the excitation of the polar oscillation modes of the gravastar. For ultracompact gravastars the frequency of these peaks depends mildly on the gravastar compactness. For masses $M \sim 10^{6} M_{\odot}$ the peaks typically lie within the optimal sensitivity bandwidth of the Laser Interferometer Space Antenna, potentially providing a unique signature of the horizonless nature of the central object. For relatively modest values of the gravastar compactness the radiated power has even more peculiar features, carrying the signature of the microscopic properties of the physical surface replacing the event horizon.
\end{abstract}

DOI: $10.1103 /$ PhysRevD.81.084011

PACS numbers: 04.30.Db, 04.25.Nx, 04.80.Cc

\section{INTRODUCTION}

One of the most elusive properties characterizing the black hole $(\mathrm{BH})$ solutions of general relativity is the presence of their event horizon. Traditional electromagnetic astronomy can at best yield lower limits on the gravitational redshift corresponding to hypothetical surfaces replacing the event horizon (see [1-4] for different viewpoints on this delicate issue). However, present and planned gravitational wave (GW) detectors offer new prospects for "directly" observing BHs and probing their structure [5].

From a gravitational point of view the structure of compact objects is encoded in their free oscillation spectrum, i.e. in their quasinormal modes (QNMs) $[6,7]$. In an effort to point out the peculiar features of compact objects whose external metric is identical to the Schwarzschild metric, but which do not possess an event horizon, in Ref. [8] (henceforth paper I) we studied the free oscillations of one of the simplest ultracompact horizonless objects: a nonrotating thin-shell gravastar. Our analysis completed previous investigations $[9,10]$ by considering the thin shell as a dynamical entity. The QNM spectrum of a thin-shell gravastar is complex and profoundly different from that of a BH, mainly because of the different boundary conditions at the surface replacing the event horizon.

\footnotetext{
*paolo.pani@ca.infn.it

†berti@phy.olemiss.edu

vitor.cardoso@ist.utl.pt

§yanbei@tapir.caltech.edu

"norte@caltech.edu
}

As first proposed by Ryan [11,12], an exquisite map of $\mathrm{BH}$ spacetimes can be constructed by observing the gravitational waveform emitted when a small compact object spirals into the putative massive $\mathrm{BH}$ at the center of a galaxy with the Laser Interferometer Space Antenna (LISA). Li and Lovelace refined the analysis showing that the small object's tidal coupling encodes additional information about the metric of the spacetime [13]. In this paper we use the formalism developed in paper I to show that any surface replacing the BH event horizon will produce a very specific signature in the gravitational signal emitted by the orbiting object because of the resonant scattering of gravitational radiation, which can be traced back to the different QNM spectrum of the two objects. In fact, here we show that the QNMs of ultracompact thinshell gravastars can be excited during the inspiral, whereas Schwarzschild QNMs can only be excited by particles plunging into the $\mathrm{BH}$ (see Refs. [14-17] for a discussion of the analogous problem of particles orbiting neutron stars).

This work is very similar in spirit to a previous study by Kesden and collaborators [18]. There are two main differences between our work and theirs. The first difference is that Kesden et al. considered boson stars rather than gravastars as BH strawmen, so no "hard surface" replaces the event horizon in their case. The second difference is that we compute the radiation in a consistent perturbative framework, instead of using "kludge" waveforms that become increasingly inaccurate in the relativistic regime. In this sense, this paper is the first "strong-field" calculation of the potential gravitational signatures of inspirals into horizonless objects. 
The plan of the paper is as follows. In Sec. II A we review the equations describing axial and polar perturbations in the interior of a gravastar and the matching conditions with the ordinary perturbations of a Schwarzschild metric in the exterior spacetime. In Sec. II B we summarize the perturbed Einstein equations in the Bardeen-PressTeukolsky (BPT) formalism and we write down the source term appearing on the right-hand side of the BPT equation for orbiting pointlike particles of mass $m_{0} \ll M$. Then we discuss how the perturbation functions outside the shell (as obtained by the matching conditions derived in paper I) can be used to solve numerically the BPT equation with a source given by the orbiting particle and to compute the radiated power. In Sec. III we compare numerical calculations of the power radiated by BHs and different gravastar models and we stress potentially observable GW signatures of horizonless ultracompact objects. We conclude by discussing possible extensions of our work.

\section{GRAVITATIONAL PERTURBATIONS BY A POINT PARTICLE}

The emission of gravitational waves by an extreme mass ratio binary system can be computed by a perturbative approach. One of the two compact objects is assumed to be an extended body of mass $M$, whose equilibrium structure is described by an exact solution of the Einstein equations. The second object is regarded as a point particle of mass $m_{0} \ll M$ perturbing the gravitational field of the companion. This method has been applied to study gravitational radiation from particles orbiting BHs (see e.g. [1921]) and neutron stars [14-16]. Here we apply the same perturbative approach to compute the power radiated by particles orbiting a thin-shell gravastar.

\section{A. Gravitational perturbations of the internal structure}

In this and the following sections we shall use the same notation as in paper I. We consider a static thin-shell gravastar with metric $[10,22]$

$$
d s_{0}^{2}=-f(r) d t^{2}+\frac{1}{h(r)} d r^{2}+r^{2}\left(d \theta^{2}+\sin ^{2} \theta d \varphi^{2}\right),
$$

with

$$
f(r)= \begin{cases}h=1-\frac{2 M}{r}, & r>a, \\ \alpha h=\alpha\left(1-\frac{8 \pi \rho}{3} r^{2}\right), & r<a,\end{cases}
$$

where $M$ is the gravastar mass and $\rho=3 M /\left(4 \pi a^{3}\right)$ is the "energy density" of the interior region. The junction conditions require the induced metric to be continuous across the shell at $r=a$. This implies that $f(r)$ must also be continuous at $r=a$, i.e. $\alpha=1$. In order to compute the radiation emitted by a gravastar perturbed by a massive point particle we must compute the gravitational perturbations both inside and outside the gravastar. Perturbations in the interior have been discussed in paper I using the ReggeWheeler (RW) gauge. Here we only recall some results that are useful to compute the power radiated by orbiting particles.

In the de Sitter interior, both axial and polar perturbations can be reduced to the study of the master equation

$$
\frac{d^{2} \Psi^{\text {in }}}{d r_{*}^{2}}+\left[\omega^{2}-\frac{l(l+1)}{r^{2}} f(r)\right] \Psi^{\text {in }}=0,
$$

where the tortoise coordinate $r_{*}$ is defined by $d r / d r_{*}=$ $f(r)$ and $f(r)$ is given by Eq. (2.2) with $r<a$. The regular solution at the center $(r=0)$ is

$$
\Psi^{\text {in }}=r^{l+1}\left(1-C(r / 2 M)^{2}\right)^{-i(2 M \omega / 2 \sqrt{C})} F\left(\frac{l+2-i \frac{2 M \omega}{\sqrt{C}}}{2}, \frac{1+l-i \frac{2 M \omega}{\sqrt{C}}}{2}, l+\frac{3}{2}, C(r / 2 M)^{2}\right),
$$

where $C \equiv(2 M / a)^{3}=8 \mu^{3}$ and $F(a, b, c, z)$ is the hypergeometric function [23]. From $\Psi^{\text {in }}$ and its derivative we can obtain the Zerilli and RW perturbation functions as explained in paper I.

For a thin-shell gravastar the background surface energy vanishes, $\Sigma=0$, but the surface stress-energy tensor $\Theta$ is, in general, nonvanishing. This implies that the perturbation functions are discontinuous across the shell. In paper I we derived the matching conditions relating interior and exterior perturbations in the RW gauge. For axial perturbations these matching conditions read

$$
\left[\left[h_{0}\right]\right]=0, \quad\left[\left[\sqrt{h} h_{1}\right]\right]=0,
$$

where $[[\ldots]]$ denotes the "jump" of a given quantity across the shell, i.e. the difference between the limits of the corresponding quantity as $r \rightarrow a_{ \pm}$. For a thin-shell grav- astar, Eqs. (2.5) imply continuity of the RW function and its derivative across the shell. The treatment of polar perturbations is more involved and it yields the following relations for the jump of the polar metric functions across the shell:

$$
\begin{gathered}
{[[K]]=0, \quad\left[\left[K^{\prime}\right]\right]=-8 \pi \frac{\delta \Sigma}{\sqrt{f(a)}},} \\
\frac{2 M}{a^{2}}[[H]]-\left[\left[H f^{\prime}\right]\right]-2 f(a)\left[\left[H^{\prime}\right]\right]+4 i \omega\left[\left[H_{1}\right]\right] \\
=16 \pi \sqrt{f(a)}\left(1+2 v_{s}^{2}\right) \delta \Sigma .
\end{gathered}
$$

The parameter $v_{s}$ depends on the equation of state (EOS) on the thin shell, $\Theta=\Theta(\Sigma)$ : 


$$
v_{s}^{2} \equiv-\left(\frac{\partial \Theta}{\partial \Sigma}\right)_{\Sigma=0},
$$

and it has the dimensions of a velocity. A microscopic model of matter on the thin shell is needed for a microphysical interpretation of $v_{s}$, but (roughly speaking) this parameter is related to the sound speed on the shell. We shall follow the treatment in paper I and treat $v_{s}$ as a free parameter, although we will primarily focus on the (presumably more physical) range $0<v_{s}^{2}<1$.

\section{B. The source term and the BPT formalism}

A detailed treatment of the perturbative approach used to compute the gravitational emission by a particle orbiting a polytropic neutron star can be found in Refs. [15-17,24]. Here we review the original method with an emphasis on the modifications required to deal with thin-shell gravastars.

The radial part $\Psi_{l m}(\omega, r)$ of the perturbation of the Weyl scalar $\delta \Psi_{4}$ is defined as

$$
\begin{aligned}
\Psi_{l m}(\omega, r)= & \frac{1}{2 \pi} \int d \Omega d t_{-2} S_{l m}^{*}(\theta, \phi) \\
& \times\left[r^{4} \delta \Psi_{4}(t, r, \theta, \phi)\right] e^{i \omega t},
\end{aligned}
$$

where $-2 S_{l m}(\theta, \phi)$ is a spin-weighted spherical harmonic of spin -2 . The function (2.8) can be expressed in terms of the Zerilli and RW perturbation functions $\left(Z_{l}(\omega, r)\right.$ and $Y_{l}(\omega, r)$, respectively) as follows:

$$
\begin{aligned}
\Psi_{l m}(\omega, r)= & \frac{r^{3} \sqrt{n(n+1)}}{4 \omega}\left[V^{\mathrm{ax}} Y_{l}+\left(W^{\mathrm{ax}}+2 i \omega\right) \Lambda_{+} Y_{l}\right] \\
& -\frac{r^{3} \sqrt{n(n+1)}}{4}\left[V^{\mathrm{pol}} Z_{l}+\left(W^{\mathrm{pol}}+2 i \omega\right) \Lambda_{+} Z_{l}\right],
\end{aligned}
$$

where $\quad 2 n=(l-1)(l+2), \quad \Lambda_{+}=d / d r_{*}+i \omega=$ $r^{-2} \Delta d / d r+i \omega$, and

$$
\begin{gathered}
W^{\mathrm{ax}}=\frac{2}{r^{2}}(r-3 M), \\
W^{\mathrm{pol}}=2 \frac{n r^{2}-3 M n r-3 M^{2}}{r^{2}(n r+3 M)} .
\end{gathered}
$$

The functions $V^{\mathrm{pol}}$ and $V^{\mathrm{ax}}$ are the well-known Zerilli and RW potentials

$$
V^{\mathrm{ax}}(r)=f\left(\frac{l(l+1)}{r^{2}}-\frac{6 M}{r^{3}}\right)
$$

$$
V^{\mathrm{pol}}(r)=\frac{2 f}{r^{3}}\left(\frac{9 M^{3}+3 \lambda^{2} M r^{2}+\lambda^{2}(1+\lambda) r^{3}+9 M^{2} \lambda r}{(3 M+\lambda r)^{2}}\right),
$$

with $\lambda=l(l+1) / 2-1$.

The radial part of $\delta \Psi_{4}$ outside the shell can be computed by solving the perturbation equations in the interior. In Refs. [15-17,24] this has been done for polytropic neutron stars by numerically integrating the Tolman-OppenheimerVolkoff equation together with the relevant stellar perturbation equations. As discussed in Sec. II A, for thin-shell gravastars the background spacetime and the perturbation functions in the interior are known analytically. Using the matching conditions derived in paper I we can determine the jump in the perturbation functions across the shell. Therefore we can easily construct the function $\delta \Psi_{4}\left(a_{+}\right)$ outside the shell. Through the Fourier and angular expansion of Eq. (2.8) we can now impose boundary conditions at $a_{+}$for the integration of the inhomogeneous BPT equation $[25,26]$

$$
\begin{aligned}
\mathcal{L}_{\mathrm{BPT}} \Psi_{l m}(\omega, r) \equiv & \left\{\Delta^{2} \frac{d}{d r}\left[\frac{1}{\Delta} \frac{d}{d r}\right]\right. \\
& +\left[\frac{\left(r^{4} \omega^{2}+4 i(r-M) r^{2} \omega\right)}{\Delta}\right. \\
& -8 i \omega r-2 n]\} \Psi_{l m}(\omega, r)=-T_{l m}(\omega, r),
\end{aligned}
$$

where $\Delta=r^{2}-2 M r$ and the source term $T_{l m}(\omega, r)$ describes the point mass $m_{0}$ moving on a given orbit around the gravastar. In Ref. [15] the solution of Eq. (2.14) is constructed in the general case of elliptic orbits. Eccentricity is expected to play an important role in extreme mass ratio inspirals $[27,28]$. However, in the remainder of this paper we focus on circular inspirals. This simplifies our study and it is sufficient to prove our main point: the gravitational radiation from extreme mass ratio inspirals around horizonless objects is drastically different from the $\mathrm{BH}$ case. We mention in passing that our numerical code is capable of handling eccentric orbits, and the extension of our study to eccentric inspirals could be an interesting topic for future research.

We further simplify the problem by using the so-called adiabatic approximation (i.e. we assume that the radiation reaction time scale is much longer than the orbital time scale). Under this assumption the trajectory of the particle is described by the geodesic equations for a mass $m_{0}$ moving on a circular orbit of radius $R_{0}$ :

$$
\bar{\gamma} \equiv \frac{d t}{d \tau}=\frac{E}{1-\frac{2 M}{R_{0}}}, \quad \omega_{\mathrm{K}} \equiv \frac{d \varphi}{d t}=\frac{1}{\bar{\gamma}} \frac{d \varphi}{d \tau},
$$

where $\tau$ is the proper time, $E$ is the energy per unit mass of the particle, and $\omega_{\mathrm{K}}=\sqrt{M / R_{0}^{3}}$ denotes the Keplerian or- 
bital frequency. The source term can be written as

$$
\begin{aligned}
T_{l m}(\omega, r)= & \delta\left(\omega-m \omega_{K}\right)\left[S_{0}^{*}\left(\frac{\pi}{2}, 0\right)_{0} U_{l m}\right. \\
& +{ }_{-1} S_{l m}^{*}\left(\frac{\pi}{2}, 0\right)_{-1} U_{l m} \\
& \left.+{ }_{-2} S_{l m}^{*}\left(\frac{\pi}{2}, 0\right)_{-2} U_{l m}\right]
\end{aligned}
$$

where the functions $s U_{l m}$ are explicitly given in Refs. [16,24].

The solution of Eq. (2.14) satisfying the boundary conditions of pure outgoing radiation at radial infinity and matching continuously with the interior solution can be found by the Green's functions technique. The amplitude of the wave at radial infinity can be shown to be [15]

$$
A_{l m}(\omega)=-\frac{1}{W_{l m}(\omega)} \int_{R}^{\infty} \frac{d r^{\prime}}{\Delta^{2}} \Psi_{l m}^{1}\left(\omega, r^{\prime}\right) T_{l m}\left(\omega, r^{\prime}\right),
$$

where $W_{l m}(\omega)$ is the Wronskian of the two independent solutions of the homogeneous BPT equation

$$
W_{l m}(\omega)=\frac{1}{\Delta}\left[\Psi_{l m}^{1} \partial_{r} \Psi_{l m}^{0}-\Psi_{l m}^{0} \partial_{r} \Psi_{l m}^{1}\right] .
$$

The two solutions $\Psi_{l m}^{0}$ and $\Psi_{l m}^{1}$ satisfy different boundary conditions:

$$
\begin{aligned}
& \mathcal{L}_{\mathrm{BPT}} \Psi_{l m}^{0}(\omega, r)=0, \quad \Psi_{l m}^{0}(\omega, r \rightarrow \infty)=r^{3} e^{\mathrm{i} \omega r_{*}}, \\
& \mathcal{L}_{\mathrm{BPT}} \Psi_{l m}^{1}(\omega, r)=0, \quad \Psi_{l m}^{1}(\omega, a)=\bar{\Psi}_{l m}(\omega, a), \\
& \partial_{r} \Psi_{l m}^{1}(\omega, a)=\partial_{r} \bar{\Psi}_{l m}(\omega, a) .
\end{aligned}
$$

Here $\mathcal{L}_{\mathrm{BPT}}$ is the differential operator on the left-hand side of the BPT Eq. (2.14) and $\bar{\Psi}_{l m}(\omega, a)$ is the radial perturbation of the Weyl scalar, constructed according to Eq. (2.9) in terms of the perturbed metric functions in the interior and evaluated at the (exterior) surface of the gravastar. The crucial point here is that the boundary conditions at the shell of a gravastar are drastically different from the ingoing-wave boundary conditions that must be imposed at the horizon of a black hole. As discussed in paper I, perturbations near the shell will in general contain a combination of ingoing and outgoing waves, even when the compactness of the gravastar approaches the Schwarzschild value $(\mu \rightarrow 1 / 2)$.

The integral in Eq. (2.17) can be written in terms of $\Psi_{l m}^{1}$ and its derivatives [16]. In Eq. (2.17) it is convenient to isolate the contribution of the Dirac $\delta$ function:

$$
A_{l m}(\omega)=m_{0} \hat{A}_{l m}(\omega) \delta\left(\omega-m \omega_{K}\right) .
$$

Then the time-averaged energy flux

$$
\begin{aligned}
\dot{E}^{R} & \equiv\left\langle\frac{d E_{\mathrm{GW}}}{d t}\right\rangle=\lim _{T \rightarrow \infty} \frac{E_{\mathrm{GW}}}{T} \\
& =\lim _{T \rightarrow \infty} \frac{1}{T} \sum_{l m} \int d \omega\left(\frac{d E_{\mathrm{GW}}}{d \omega}\right)_{l m}
\end{aligned}
$$

can be written in terms of $\hat{A}_{l m}(\omega)$ as follows:

$$
\dot{E}^{R}\left(m \omega_{K}\right)=\sum_{l m} \frac{m_{0}^{2}}{4 \pi\left(m \omega_{K}\right)^{2}}\left|\hat{A}_{l m}\left(m \omega_{K}\right)\right|^{2} \equiv \sum_{l m} \dot{E}_{l m}^{R} .
$$

In order to evaluate $\Psi_{l m}^{0}$ and $\Psi_{l m}^{1}$, we integrate the BPT equation by an adaptive Runge-Kutta method. Close to a resonance the solutions must be computed very accurately, since the Wronskian (2.18) is the difference between two terms that almost cancel each other. When required, the tolerance parameter in the adaptive integration routines is decreased to achieve convergence. Since the orbital frequency is related to the orbital velocity $v$ and to the semilatus rectum (which for circular orbits is simply $\left.p=R_{0} / M\right)$ by the relations

$$
v=\left(M \omega_{K}\right)^{1 / 3}=p^{-1 / 2},
$$

the energy flux $\dot{E}^{R}$ can also be considered as a function of $v$ or $p$. In the following we shall normalize $\dot{E}^{R}$ to the Newtonian quadrupole energy flux

$$
\dot{E}^{N}=\frac{32}{5} \frac{m_{0}^{2}}{M^{2}} v^{10} .
$$

Then the energy flux emitted in gravitational waves normalized to the Newtonian quadrupole energy flux is given by

$$
P(v) \equiv \frac{\dot{E}^{R}}{\dot{E}^{N}}=\sum_{l m} \frac{5}{128 \pi} \frac{M^{2}}{\left(m \omega_{K}\right)^{2} v^{10}}\left|\hat{A}_{l m}\left(m \omega_{K}\right)\right|^{2} .
$$

The normalized energy flux (2.25) can be computed up to $v \leq 1 / \sqrt{6} \simeq 0.408$, which corresponds to the innermost stable circular orbit (ISCO) at $R_{0}=6 M$. The postNewtonian expansion of the energy flux $P(v)$ for particles in circular orbit around Schwarzschild BHs has been studied by several authors $[21,29,30]$. The instability of circular orbits with $R_{0}<6 M$ sets an upper bound on the velocity of the point mass. If the radius of the gravastar is larger than the ISCO (this typically occurs for $\mu<0.1666$ ) the upper limit in $v$ will be smaller.

From the analytical form of the stress-energy tensor (2.16) it is easy to see that, for each assigned $l$, a mode of the star is excited when the orbital frequency satisfies the resonant condition

$$
m \omega_{\mathrm{K}}=\omega_{\mathrm{QNM}},
$$

where $\omega_{\mathrm{QNM}}$ is the QNM frequency. Thus we expect sharp peaks to appear at the values of $v$ corresponding to the excitation of the gravastar QNMs for different values of the 
angular momentum parameter $l$. This offers an intriguing signature of the absence of event horizons, since the emitted power for a Schwarzschild BH does not show any peak. In fact one can easily check that the frequency of the fundamental QNM of a Schwarzschild BH is higher than the critical value $m \omega_{\mathrm{K}}$ corresponding to a particle at the ISCO [24]. In other words, Schwarzschild QNMs can only be excited by particles plunging into the $\mathrm{BH}$, while the QNMs of a gravastar can be excited during the inspiral. In the following section we will compare the power emitted by a circular inspiral around a thin-shell gravastar to the power emitted by a circular inspiral around a Schwarzschild BH.

\section{GRAVITATIONAL FLUX FROM GRAVASTARS AND BLACK HOLES}

Thin-shell gravastar models are specified by two parameters: the gravastar compactness $\mu=M / a$ and the sound speed parameter $v_{s}$ that characterizes the EOS on the shell. Thin-shell gravastars are only one of the several possible models that can be explored (see e.g. [10,22]) but we expect the qualitative results of our analysis to apply quite in general. The reason is that the main difference between gravastars and BHs comes from the different boundary conditions at the "surface" replacing the $\mathrm{BH}$ event horizon, rather than from the specific nature of this surface. Furthermore, as discussed below, peaks in the energy flux are more sensitive to the "global" properties of the gravastar (as determined by the compactness parameter $\mu$ ) than to the microphysical model determining the matter distribution on the shell (which in our simplified case reduces to the specification of a value for $v_{s}$ ). Our numerical study covers the whole range in compactness $(0<\mu<0.5)$. We mainly focused on the most physical range of the EOS parameter $\left(0<v_{s}^{2}<1\right)$ but we also studied the superluminal case $\left(v_{s}^{2}>1\right)$, and we even allowed for models with $v_{s}^{2}<0$ [31].

The gravitational emission of a Schwarzschild BH perturbed by a particle has been studied analytically and numerically in great detail for both circular and eccentric orbits [19-21,32]. Our purpose here is to compare and contrast the energy flux from particles orbiting Schwarzschild BHs to the energy flux from particles orbiting thin-shell gravastars. For each value of the gravastar parameters $\left(\mu, v_{s}^{2}\right)$ we integrate the perturbation equations (as described in Sec. II) for a pointlike object of mass $m_{0}$ moving on a circular orbit of radius $R_{0}$ with orbital velocity $v$ and we compute the energy flux (2.25). Our numerical work uses a modified version of the BPT code described in Ref. [16]. The results obtained by the BPT formalism were verified using an independent code that integrates the Zerilli and Regge-Wheeler equations. A slight variant of these codes was used to compute the flux from a particle orbiting Schwarzschild BHs. The results are consistent with Refs. [19,21] within an accuracy of about one part in $10^{6}$ (see Ref. [30] for more details).

From the results of paper I, in the Schwarzschild limit $\mu \rightarrow 0.5$ the real part of the QNM frequency tends to zero and to a very good approximation it is independent of $v_{s}$. For example, for $\mu=0.49999$ and $l=2$ we varied $v_{s}^{2}$ in the range $[-2,2]$ in steps of $\delta v_{s}=0.1$ and we found that the real part of the modes is a constant within a part in $10^{6}$ $\left(\omega_{R}=0.235932\right)$, while the imaginary part has tiny variations in the range between $\omega_{I}=4.20 \times 10^{-7}$ and $\omega_{I}=$ $4.17 \times 10^{-7}$. In order for a QNM to be excited by particles in circular orbits, the QNM frequency must be small enough to allow for the resonant condition (2.26).

Figure 1 shows the dominant $(l=2)$ contribution to the energy flux for gravastars with very high compactness. The frequencies of the lowest QNMs of a Schwarzschild BH are higher than those of an ultracompact gravastar, and cannot be excited by particles in stable circular orbits. For this reason the power emitted by a BH (on the scale of this plot) is roughly constant. Resonance peaks do appear for gravastars, as expected, when $\omega_{\mathrm{QNM}}=2 \omega_{\mathrm{K}}$. Notice that these resonances are extremely narrow and they would get even narrower for $l>2$. This is because the imaginary part of the excited modes is extremely small $\left(2 M \omega_{I} \sim 10^{-7}\right.$, $10^{-10}$ for $l=2$ and $l=3$ respectively) in the highcompactness limit $\mu \rightarrow 0.5$, leading to a corresponding decrease in the quality factor of the oscillations. Whether these resonances are actually detectable is an interesting question for LISA data analysis. The answer depends on dissipative mechanisms (besides gravitational radiation damping) that could affect the time scale of the oscillations, especially in the nonlinear regime: see e.g. [17,33] for discussions of this problem in the context of neutron star binary detection by Earth-based GW interferometers.

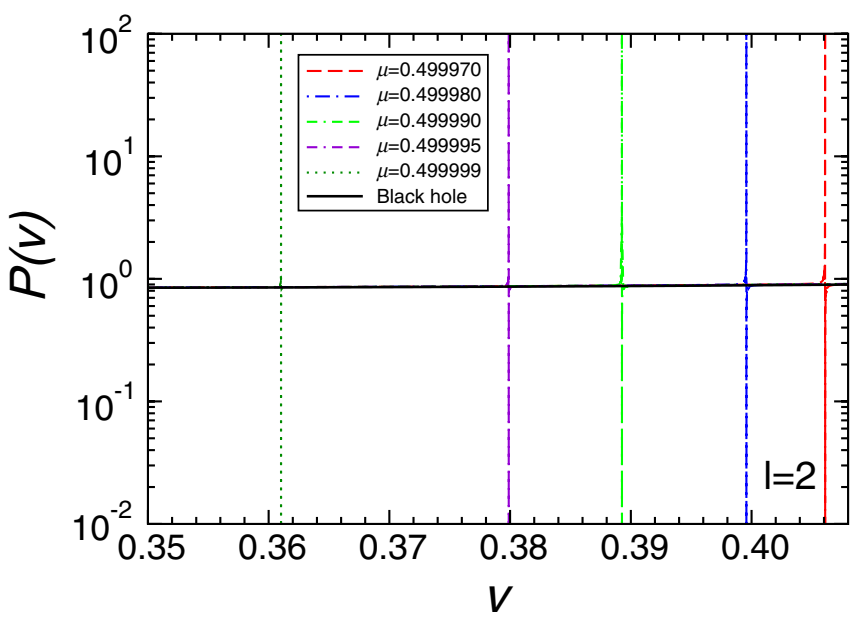

FIG. 1 (color online). Dominant $(l=2)$ contribution to the energy flux for very high compactness and $v_{s}^{2}=0.1$ (but when $\mu \sim 0.5$ resonances are almost independent on $v_{s}^{2}$ ). From right to left the resonant peaks correspond to $\mu=0.49997,0.49998$, 0.499 99, 0.499 995, 0.499 999, respectively. 
TABLE I. Values of the compactness $\mu$, angular momentum number $l$, QNM frequency, orbital velocity $v$, and GW frequency $\nu_{\mathrm{GW}}$ of the circular orbits which would excite the fundamental QNM of the gravastar for the given multipole. The Keplerian frequency is given in $\mathrm{mHz}$ and rescaled to a gravastar mass $M_{6}=10^{6} M_{\odot}$.

\begin{tabular}{lcccc}
\hline \hline$\mu$ & $l$ & $M \omega_{\mathrm{QNM}}$ & $v$ & $\left(M_{6} / M\right) \nu_{\mathrm{GW}}(\mathrm{mHz})$ \\
\hline 0.49997 & 2 & 0.1339 & 0.4061 & 4.328 \\
& 3 & 0.1508 & 0.3691 & 4.873 \\
0.49998 & 2 & 0.1276 & 0.3996 & 4.123 \\
& 3 & 0.1429 & 0.3625 & 4.616 \\
0.49999 & 2 & 0.1180 & 0.3893 & 3.812 \\
& 3 & 0.1310 & 0.3521 & 4.232 \\
0.499995 & 2 & 0.1096 & 0.3799 & 3.543 \\
0.499999 & 2 & 0.0941 & 0.3610 & 3.041 \\
\hline \hline
\end{tabular}

Quite interestingly, the gravastars that "try harder" to look like a BH (in the sense that their shell is closer to the Schwarzschild event horizon) are also those that give away their identity at smaller angular velocities: the more compact the gravastar, the smaller the peak frequency in the energy flux. Table I lists the expected excited modes for different values of $\mu$ corresponding to ultracompact gravastars.

One may worry that the resonance will eventually get out of the LISA band for gravastars having $\mu$ extremely close to the Schwarzschild value. The following naive argument suggests that this is not the case. The "thickshell gravastar" model by Mazur and Mottola predicts a microscopic but finite shell thickness $\ell \sim \sqrt{L_{\mathrm{Pl}} r_{\mathrm{S}}} \simeq 3 \times$ $10^{-14}\left(M / M_{\odot}\right)^{1 / 2} \mathrm{~cm}$, where $L_{\mathrm{Pl}}$ is the Planck scale and $r_{\mathrm{S}}$ is the Schwarzschild radius, so that the energy density and pressure in the shell are far below Planckian and the geometry can still be described reliably by Einstein's equations [34]. Our simplified model does not allow for a finite thickness of the shell, and a microscopic model of finite shells is required for a careful analysis of this problem. However, for the sake of argument, let us consider $\epsilon=1 / 2-\mu \rightarrow 0$ as a "thickness parameter" describing how far the gravastar shell can be relative to the $\mathrm{BH}$ horizon. A power-law fit of the QNMs of a thin-shell gravastar in the limit $\epsilon \rightarrow 0$ yields $f_{\mathrm{GW}} \sim 3.828 \times(\epsilon \times$ $\left.10^{-5}\right)^{0.1073}$. The lower frequency sensitivity limit for LISA is dictated by acceleration noise. Assuming lower frequency cutoffs of $f_{\text {low }}=10^{-5}, 3 \times 10^{-5}, 10^{-4}$, we find that the peaks will sweep out of the LISA band when $\epsilon=$ $9.6 \times 10^{-48}, 2.7 \times 10^{-43}, 2.0 \times 10^{-38}$, respectively. This estimate of the "minimum measurable deviation from a BH" is admittedly very sensitive to the fitting function we use and it may change when one considers thick-shell gravastars, but it suggests that LISA has the potential to reveal solid surfaces replacing horizons even when these solid surfaces are very close to the location of the Schwarzschild horizon.

A relevant question is whether massive horizonless objects which are compact by the standard of (say) main sequence stars, but "only" as compact as neutron stars, can leave a signature on the gravitational signal emitted by small, inspiraling compact objects. In Fig. 2 we plot the normalized energy flux $P(v)$ as a function of the orbital velocity for gravastar models with $v_{s}^{2}=0.1$ and compactness in the range $0.1 \lesssim \mu \lesssim 0.49$, as well as for a Schwarzschild BH. The total flux was computed by adding all multipoles $(|m| \leq l)$ and by truncating the multipolar expansion at $l=6$. As discussed in Refs. [15,24,30,32], a multipole of order $l$ contributes to the total power as a correction of order $p^{2-l}$. Roughly speaking, a truncation at
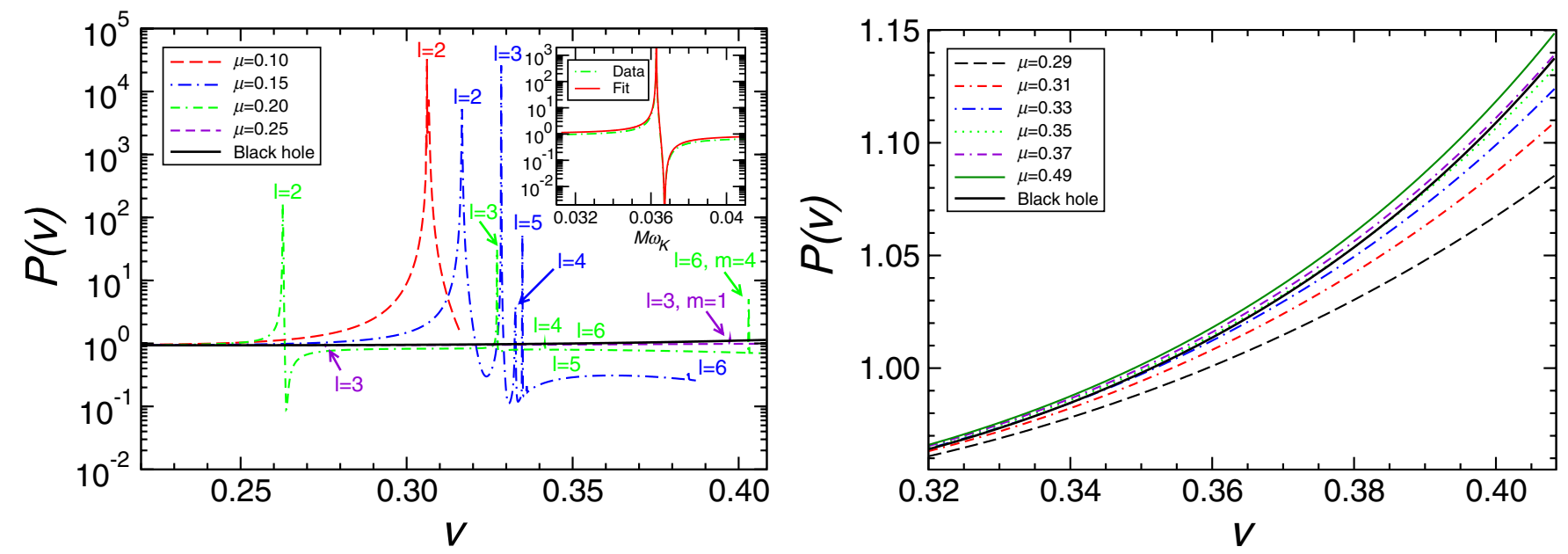

FIG. 2 (color online). Left: The energy flux (summed up to $l=6$ ) of GWs emitted by a small mass orbiting a thin-shell gravastar with $v_{s}^{2}=0.1$ and different values of $\mu$ (plotted as a function of the particle orbital velocity $v$ ) is compared with the flux for a Schwarzschild BH. All peaks (with the exception of the last two peaks on the right) are due to the excitation of QNMs with $l=m$. Right: same for $v_{s}^{2}=0.1$ and selected values of $\mu \in[0.29,0.49]$. No QNMs are excited in this range. 
$l=6$ produces a relative error (in the nonresonant regime) of order $p^{-5}=v^{10}$ (but see [30] for a more careful discussion of the convergence properties of the postNewtonian series). When $\mu \gtrsim 0.166$ the ISCO is located outside the gravastar and we plot the energy flux up to the ISCO velocity $v_{\text {ISCO }} \simeq 0.408$ (corresponding to $R_{0}=6 M$ ). For less compact gravastars, plots of the energy flux are truncated at the velocity corresponding to the location of the shell.

The complex structure of the spectrum for values of $\mu$ smaller than about 0.2 is best understood by considering the real and imaginary parts of the weakly damped QNM frequencies of a gravastar (see Fig. 3). For clarity in Fig. 3 we only plot weakly damped QNMs, but our general arguments apply also to the second, "ordinary" family of QNMs (cf. paper I). In particular, from Fig. 2 and Fig. 6 in paper I it should be clear that QNMs will be excited for low values of the compactness and when $\mu$ is very close to the $\mathrm{BH}$ value $\mu=1 / 2$. Besides these "ultracompact" modes, only QNMs whose real part lies below the horizontal line in the left panel (corresponding to twice the ISCO orbital frequency for a particle in circular orbit) can be excited.

Figure 3 clarifies that the range of $\mu$ over which QNMs can be excited depends on $v_{s}$. For $v_{s}^{2}=0.1$ (the case considered to produce the energy fluxes of Fig. 2) QNM frequencies that can be excited by resonant inspirals only exist for $\mu \lesssim 0.21$ (left panel of Fig. 2) or for $\mu \gtrsim$ 0.49997 , i.e. when the thin shell is extremely close to the location of the BH horizon (Fig. 1). The real part of the corresponding QNM frequency has a local maximum at $\mu \approx 0.15$. Correspondingly, the $l=2$ QNM peak visible in the energy flux of Fig. 2 occurs later in the inspiral for the $\mu=0.15$ model than it does for the $\mu=0.10$ and $\mu=$ 0.20 models.

In Fig. 2 the $l=2$ and $l=3$ peaks for $\mu=0.20$ are well separated in frequency and an "antiresonance" is visible to the right of the $l=2$ resonance. The nature of this antiresonance can be explained by a simple harmonic oscillator model [16]. In the inset of the left panel of Fig. 2 we plot both the resonance and antiresonance as functions of the Keplerian orbital frequency of the particle $M \omega_{\mathrm{K}}$ for $\mu=0.2$ and $l=2$ (dashed green line). A fit using the simple harmonic oscillator model of Ref. [16] (red line) reproduces the qualitative features of both resonance and antiresonance: in this specific case the fit gives $2 M \omega_{R} \sim$ 0.07257 and $2 M \omega_{I} \sim 2 \times 10^{-6}$, while QNM calculations using the resonance method yield $2 M \omega_{R} \sim 0.07257$ and $2 M \omega_{I} \sim 4 \times 10^{-6}$.

Modes with $l>2$ are typically harder to excite because of their higher frequencies and lower quality factors. However, because of the complex "selection rules" illustrated in Fig. 3 for $l=2$, sometimes only resonances with $l>2$ will be visible. When $v_{s}^{2}=0.1$ and $\mu>0.21$ only modes with $l>2$ can be excited, and only narrow $l=3$ resonances can be seen in Fig. 2 when the compactness $\mu=0.25$ (cf. Fig. 4).

When $l=2$ the imaginary part of one QNM with $v_{s}^{2}=$ 0.1 crosses zero within our numerical accuracy at the "critical" compactness $\mu \simeq 0.21$, possibly signaling a (marginal) nonradial instability of the gravastar, and no QNMs can be excited for $0.21 \leq \mu \leq 0.49997$. In this compactness range the energy flux emitted by either the gravastar or the $\mathrm{BH}$ is mostly due to the orbital motion and it only depends on the compactness of the central object. The right panel of Fig. 2 shows that the flux emitted by a
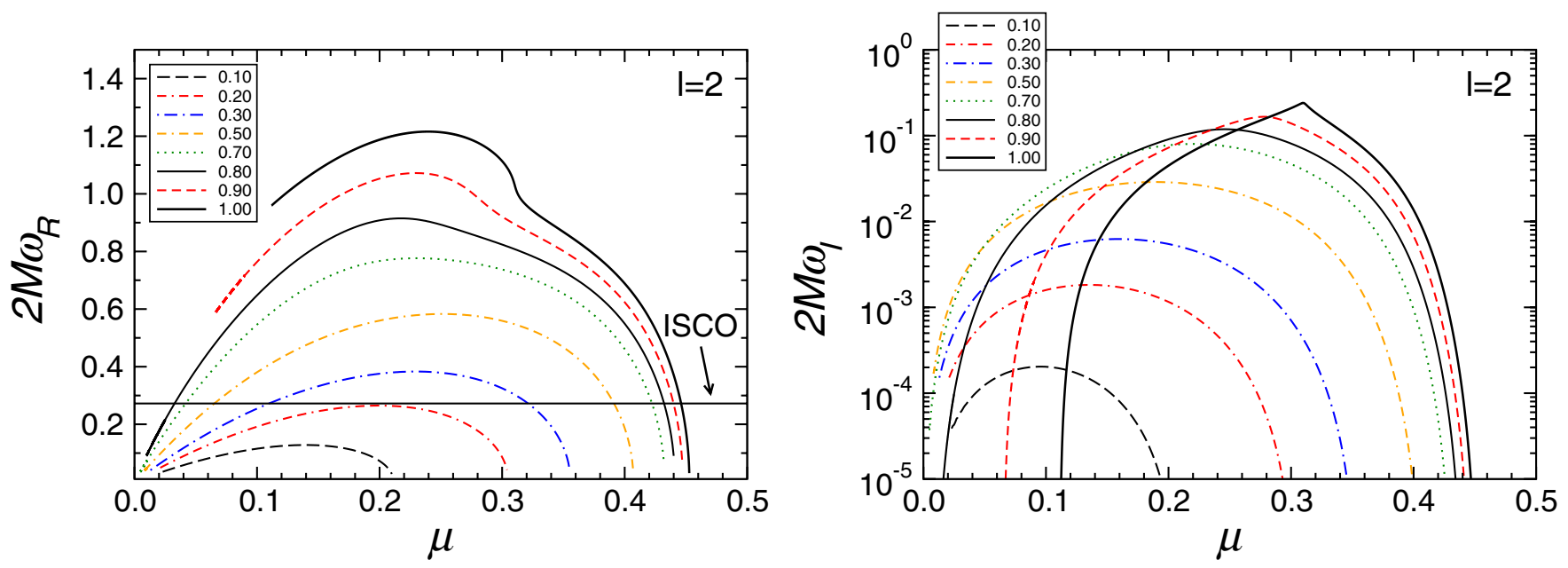

FIG. 3 (color online). Real part (left) and imaginary part (right) of gravastar QNMs with $l=2$ as a function of compactness for several fixed values of $v_{s}^{2}$ (as indicated in the legend). For clarity in illustrating the "selection rules" that determine QNM excitation during inspiral we only show the weakly damped part of the QNM spectrum (compare Fig. 2 and Fig. 6 in paper I). For $v_{s}^{2}>0.8$ the real part of the frequency is plotted down to the critical minimum compactness at which the imaginary part crosses zero within our numerical accuracy. The horizontal line at $2 M \omega_{R} \simeq 0.2722$ corresponds to twice the orbital frequency of a particle in circular orbit at the ISCO: only QNMs below this line can be excited during a quasicircular inspiral. 

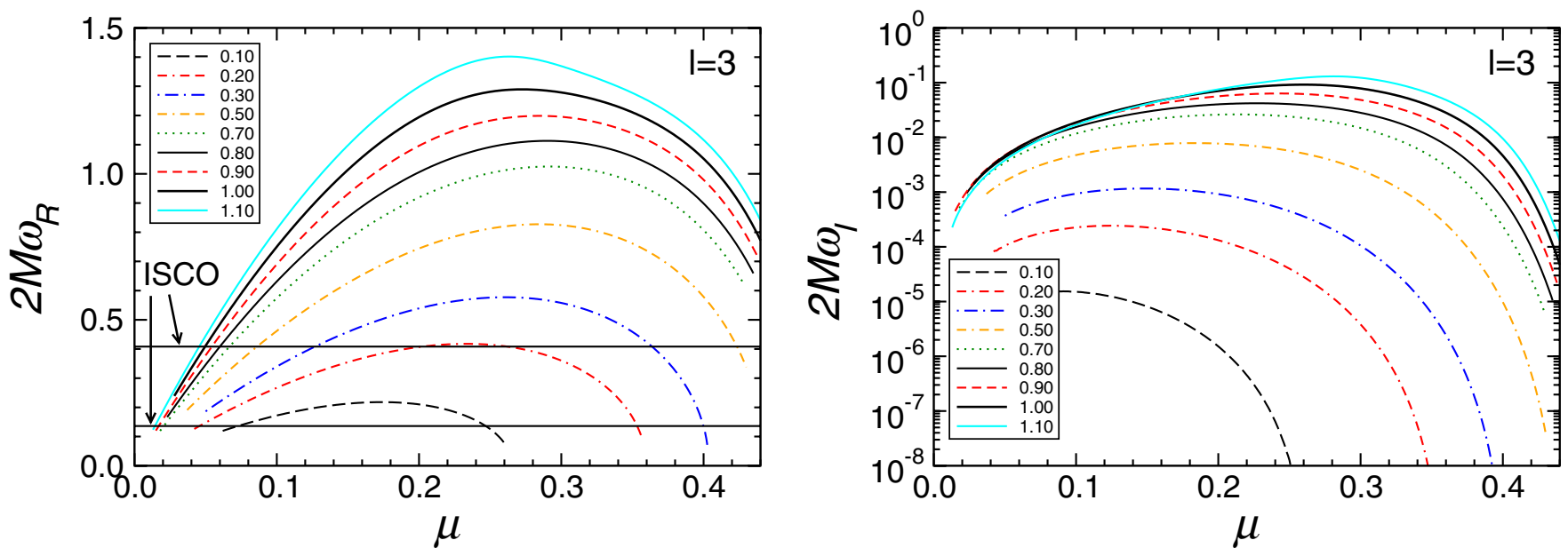

FIG. 4 (color online). Real part (left) and imaginary part (right) of gravastar QNMs with $l=3$ as a function of compactness for several fixed values of $v_{s}^{2}$ (as indicated in the legend). For clarity in illustrating the "selection rules" that determine QNM excitation during inspiral we only show the weakly damped part of the QNM spectrum. In the left panel, the horizontal lines at $2 M \omega_{R} \simeq 0.1361$ $\left(2 M \omega_{R} \simeq 0.4082\right)$ correspond to the orbital frequency (or 3 times the orbital frequency) of a particle in circular orbit at the ISCO. Perturbations with $l=3, m=1$ can excite the QNMs below the first line, while perturbations with $l=m=3$ can excite QNMs below the second line.

gravastar approaches the $\mathrm{BH}$ flux "from below" as the compactness increases. For $\mu \simeq 0.35$ the gravastar flux is almost indistinguishable from the $\mathrm{BH}$ flux and for $\mu>$ 0.35 a gravastar radiates slightly more than a $\mathrm{BH}$. This is due to the fact that the emitted power "feels" the contribution of resonances, which in this case correspond to orbits smaller than the ISCO but do nevertheless contribute to increase the slope of the curve. A similar trend can be seen in neutron star calculations in regions of the parameter space where the contribution from resonances is negligible [16].

If gravastars or other horizonless objects have astrophysical reality, the presence or absence of resonant peaks in the GW flux can provide interesting information on the microscopic properties of the physical surface replacing the event horizon. Suppose, for example, that we can estimate the compactness of a massive object by independent means (e.g. by electromagnetic observations). Even within our simple thin-shell model, the range in frequency where resonances in the GW emission from extreme mass ratio inspirals (EMRIs) are allowed changes with $v_{s}^{2}$. For example, if $v_{s}^{2}=0.1$ resonances can exist when $\mu \lesssim 0.21$ or $\mu \sim 0.5$, but if $v_{s}^{2}=0.3$ they can exist when $\mu \lesssim 0.1$, $0.33 \lesssim \mu \lesssim 0.36$, and $\mu \sim 0.5$. Similar results also hold when $l=3$, as shown in Fig. 4. For example, if $v_{s}^{2}=0.1$ resonances can exist when $\mu \lesssim 0.27$ or $\mu \sim 0.5$, but if $v_{s}^{2}=0.3$ they can exist when $\mu \lesssim 0.2,0.37 \lesssim \mu \lesssim 0.41$, and $\mu \sim 0.5$. So, in general, the range of $\mu$ where QNM frequencies can be excited by a circular inspiral depend on the value of $l$. In the Schwarzschild limit $(\mu \sim 0.5)$ QNM frequencies are excited for any $l$, but higher- $l$ modes have a tiny imaginary part $\left(2 M \omega_{I} \sim 10^{-10}\right.$ for $\left.l=3\right)$ and they are more difficult to detect than the dominant $(l=2)$ modes.
If an EMRI is detected, the existence of these selection rules (in the form of compactness regions where resonances can or cannot exist) in principle allows for null tests of the existence of an event horizon for objects of the given compactness. Similar arguments can presumably be made for more complex (or contrived) gravastar models.

We can compare the gravitational energy flux shown in Fig. 2 with that emitted by neutron stars of comparable compactness (see for instance Fig. 1 in Ref. [16]). The energy flux for a thin-shell gravastar with $\mu=0.1$ is shown in Fig. 2 by a red dashed line. From the peak position we find that the GW frequency for $l=2$ is $\nu_{\mathrm{GW}} \sim$ $1.94 \mathrm{kHz}$ for $M=0.945 M_{\odot}$. This is comparable with the value of a polytropic neutron star with the same mass and compactness, $\nu_{\mathrm{GW}} \sim 1.53 \mathrm{kHz}$ (cf. model A in Ref. [16]). These frequencies depend on the equation of state on the shell of the gravastar and in the neutron star interior, respectively. However their range is set by the total mass, so both frequencies lie within the optimal sensitivity bandwidth of the Laser Interferometer Gravitational-Wave Observatory (LIGO). Therefore even gravastars which are as compact and massive as neutron stars can leave a potentially detectable imprint in the LIGO bandwidth.

\section{CONCLUSIONS}

We have shown that gravitational radiation from EMRIs can be used to tell the presence or absence of an event horizon in a compact, massive object. More specifically, we have shown that the resonant excitation of the oscillation modes of a gravastar in the LISA band is a potentially observable signature of the surface replacing the event horizon. For thin-shell gravastar models there is a range 
of compactness (e.g. $\mu \lesssim 0.21$ and $\mu \gtrsim 0.49997$ for $v_{s}^{2}=$ 0.1 ) where this resonant scattering can occur.

More detailed data analysis studies (possibly including refined microphysical models of this "solid surface") are necessary to determine the detectability of resonant peaks, especially for ultracompact gravastars. The extension of our results to rotating gravastar models presents a challenge because of the difficulties in finding plausible rotating gravastar solutions and because of the ergoregion instability that affects some rotating gravastar models [35-37].

\section{ACKNOWLEDGMENTS}

P.P. thanks the Department of Physics, University of Rome "La Sapienza" for the kind hospitality. E. B.'s re- search was supported by NSF Grant No. PHY-0900735. V. C. was supported by a "Ciência 2007" research contract and by Fundação Calouste Gulbenkian. Y.C. was supported by NSF Grant Nos. PHY-0653653 and PHY0601459, and the David and Barbara Groce Start-up Fund at Caltech. This work was partially supported by FCT-Portugal through Project Nos. PTDC/FIS/64175/ 2006, PTDC/FIS/098025/2008, PTDC/FIS/098032/2008, PTDC/CTE-AST/098034/2008, and CERN/FP/109290/ 2009. The authors thankfully acknowledge the computer resources, technical expertise, and assistance provided by the Barcelona Supercomputing Centre-Centro Nacional de Supercomputación.
[1] R. Narayan, New J. Phys. 7, 199 (2005).

[2] D. Psaltis, arXiv:0806.1531.

[3] M. Visser, Proc. Sci., BHs,GRandStrings2008 (2008) 001 [arXiv:0901.4365].

[4] M. A. Abramowicz, W. Kluzniak, and J.-P. Lasota, Astron. Astrophys. 396, L31 (2002).

[5] B.S. Sathyaprakash and B.F. Schutz, Living Rev. Relativity 12, 2 (2009).

[6] K. D. Kokkotas and B. G. Schmidt, Living Rev. Relativity 2, 2 (1999).

[7] E. Berti, V. Cardoso, and A. O. Starinets, Classical Quantum Gravity 26, 163001 (2009).

[8] P. Pani, E. Berti, V. Cardoso, Y. Chen, and R. Norte, Phys. Rev. D 80, 124047 (2009).

[9] P. P. Fiziev, Classical Quantum Gravity 23, 2447 (2006).

[10] C. B. M. H. Chirenti and L. Rezzolla, Classical Quantum Gravity 24, 4191 (2007).

[11] F. D. Ryan, Phys. Rev. D 52, 5707 (1995).

[12] F. D. Ryan, Phys. Rev. D 56, 1845 (1997).

[13] C. Li and G. Lovelace, Phys. Rev. D 77, 064022 (2008).

[14] Y. Kojima, Prog. Theor. Phys. 77, 297 (1987).

[15] L. Gualtieri, E. Berti, J.A. Pons, G. Miniutti, and V. Ferrari, Phys. Rev. D 64, 104007 (2001).

[16] J. A. Pons, E. Berti, L. Gualtieri, G. Miniutti, and V. Ferrari, Phys. Rev. D 65, 104021 (2002).

[17] E. Berti, J.A. Pons, G. Miniutti, L. Gualtieri, and V. Ferrari, Phys. Rev. D 66, 064013 (2002).

[18] M. Kesden, J. Gair, and M. Kamionkowski, Phys. Rev. D 71, 044015 (2005).

[19] C. Cutler, E. Poisson, G. J. Sussman, and L. S. Finn, Phys. Rev. D 47, 1511 (1993).

[20] C. Cutler, D. Kennefick, and E. Poisson, Phys. Rev. D 50,
3816 (1994).

[21] E. Poisson, Phys. Rev. D 52, 5719 (1995).

[22] M. Visser and D. L. Wiltshire, Classical Quantum Gravity 21, 1135 (2004).

[23] M. Abramowitz and I. A. Stegun, Handbook of Mathematical Functions with Formulas, Graphs, and Mathematical Tables (Dover, New York, 1972).

[24] E. Berti, Ph.D. thesis, University of Rome "La Sapienza", 2002.

[25] J. M. Bardeen and W. H. Press, J. Math. Phys. (N.Y.) 14, 7 (1973).

[26] S. A. Teukolsky, Astrophys. J. 185, 635 (1973).

[27] P. Amaro-Seoane et al., Classical Quantum Gravity 24, R113 (2007).

[28] N. Yunes, K. G. Arun, E. Berti, and C. M. Will, Phys. Rev. D 80, 084001 (2009).

[29] Y. Mino, M. Sasaki, M. Shibata, H. Tagoshi, and T. Tanaka, Prog. Theor. Phys. Suppl. 128, 1 (1997).

[30] N. Yunes and E. Berti, Phys. Rev. D 77, 124006 (2008).

[31] E. Poisson and M. Visser, Phys. Rev. D 52, 7318 (1995).

[32] E. Poisson, Phys. Rev. D 47, 1497 (1993).

[33] E. E. Flanagan and T. Hinderer, Phys. Rev. D 77, 021502 (2008).

[34] P. O. Mazur and E. Mottola, Proc. Natl. Acad. Sci. U.S.A. 101, 9545 (2004).

[35] V. Cardoso, P. Pani, M. Cadoni, and M. Cavaglia, Phys. Rev. D 77, 124044 (2008).

[36] V. Cardoso, P. Pani, M. Cadoni, and M. Cavaglia, Classical Quantum Gravity 25, 195010 (2008).

[37] C. B. M. H. Chirenti and L. Rezzolla, Phys. Rev. D 78, 084011 (2008). 